of the many useless organic reagents for metals. Some advances have undoubtedly been made in the development of new reactions and reagents, but without the availability of physical methods, the determination of alkali metals would still be a major problem in many industrial and biochemical laboratories.

Every aspect of the analytical chemistry of the six metals described in Part 2, Volume 3, copper, cadmium, magnesium, mercury, tin and zinc is detailed in the same thorough way. As with the section on alkali metals, determinations of the metal in a wide range of industrial and other materials are described. It is somewhat surprising to find that the shortest sections are on copper and tin which have about forty pages, whereas mercury has about one hundred pages. Although the two shorter sections cover the ground quite well, more space should have been devoted to the subjects. For example, probably more spectrophotometric methods have been described for copper than for any other metal except iron, and a critical assessment of the more recent would have onhanced the value of this section. Only the well-known standard procedures are described. Similarly the reagent $N$-benzoyl- $N$ phenyl-hydroxylamine appears to have advantages over cupferron in the determination of tin and has been known for a sufficient number of years to warrant assessment, but only the uses of cupferron are described.

The editors and authors of these volumes deserve the highest praise for the volumes which have appeared so far. The general lay-out of each section has obviously been the subject of considerable thought and planning. Future volumes will be awaited with interest.

R. Belcher

\section{FURTHER ADVANCES IN FLUORINE CHEMISTRY}

Advances in Fluorine Chemistry

Vol. 2. Edited by Prof. M. Stacey, Prof. J. C. Tatlow and Dr. A. G. Sharpo. Pp. iv +220. (London: Butterworth and Co. (Publishers), Ltd., 1961.) $45 s$.

THE second volume in this series continues with six selected topics in fluorine chemistry.

"The Thermochemistry of Organic Fluorine Com. pounds" (C. R. Patrick) deals with those general topics associated with the thermochemistry and thermodynamics of materials. Emphasis is given to the properties of the $\mathrm{C}-\mathrm{F}, \mathrm{C}-\mathrm{H}, \mathrm{C}-\mathrm{C}$ and $\mathrm{C}-\mathrm{X}$ (halogen) bonds in organic fluorides. The problems of the dissociation energy of fluorine and the so-called thermal decomposition of fluorine-containing polymers is reviewed. The impression is made that some of the available data in those areas is still less precise than is desirable and that considerable experimental work remains to be done.

The section on "Fluorine Resources and Fluorine Utilization" (C. G. Finger) is based on a paper presented by the author at the First International Symposium on Fluorine Chemistry. It is a statistical and technological review of the current world-wide sources of the basic fluorides and their use in the manufacture of fluorine-containing products for industrial and commercial uses.

A third article is on "Mass Spectrometry of Fluorine Compounds" (J. R. Majer). This selection compiles and correlates mass spectrometric, ionization potential and appearance potential data of a large number of perfluoro and polyfluoro organic derivatives. A short section on the nitrogen and fluorides completes this exceedingly thorough presentation.

"The Fluorination of Organic Compounds Using Elementary Flourine" (J. M. Tedder) is concerned with the theoretical aspects, experimental techniques and the reactions of a large variety of organic compounds with elemental fluorine under different conditions. Work in this field in the past ten years has seen a "shift of emphasis from attempts to develop preparative methods towards a study of the course and mechanism of the (direct) fluorination process".

The main emphasis in "The Fluorides of the Actinide Elements" (W. Hodge) is on the more readily available elements, thorium, uranium and plutonium. The elements actinium, protactinium, neptunium, americium and curium are treated more briefly. The conditions for and the methods of preparation of the various fluorides and oxyfluorides are discussed. Where applicable, the physical properties of the simple and complex systems are encountered and considered in the light of theoretical considerations.

The final section, "The Physiological Action of Organic Compounds Containing Fluorine" (B. C. Saunders), considers the toxicological, neurological and therapeutic effects of a number of classes of fluorides, organic and inorganic, on plant and animal life. Antidotes are suggested for some toxic agents and the relation of structure to metabolism and other physiological effects is treated in the case of some substances. It is pointed out that many of the materials were prepared originally for use as potential chemical warfare agents, which, fortunately, were never used for that purpose, but that "an attempt has been made to explain the uses to which these compounds have been put and the applications provide a striking example of beating the proverbial swords into ploughshares".

The second volume of Advances in Fluorine Chemistry, like its predecessor, is a more than adequate publication that continues to demonstrate that the field of fluorine chemistry traverses the science of chemistry at every intersection and implies that it will continue to do so with increasing prominence. RTCHARD DRESDNER

\section{DAYLIGHT ILLUMINATION}

\section{The Science of Daylight}

By Dr. John W. T. Walsh. Pp. $\mathrm{x}+285$. (London: Macdonald and Co. (Publishers), Ltd., 1961.) $40 s$. net.

AYLIGHT technology developed initially as an aid to the settlement of rights-of-light cases in courts of law, and this origin has influenced both its methods and its character up to the present day. The preoccupations with refinement have put the science of daylight in a special enclave in the wider field of illumination technology. Architects concerned with the design of buildings for function and amenity find the nice distinctions between one theoretical treatment and another not only difficult to follow but also of little consequence to their basic need to design for good daylighting. It is fortunate, therefore, that the first attempt to take a synoptic view of the science of daylight has been made by an 